\title{
Dynamics of Pyridine Adsorption on Gold(111) Terraces in Acid Solution from in-Situ Scanning Tunneling Microscopy under Potentiostatic Control
}

\author{
G. Andreasen, M. E. Vela, R. C. Salvarezza, and A. J. Arvia* \\ Instituto de Investigaciones Fisicoquímicas Teóricas y Aplicadas (INIFTA), Sucursal 4, \\ Casilla de Correo 16, (1900) La Plata, Argentina
}

Received April 22, 1997. In Final Form: August 11, 1997ð

\begin{abstract}
The adsorption of pyridine (Py) on $\mathrm{Au}(111)$ terraces produced from aqueous $0.1 \mathrm{M} \mathrm{HClO}_{4}+10^{-3} \mathrm{M} \mathrm{Py}$ at $298 \mathrm{~K}$ was studied in the potential range $0.15 \mathrm{~V}<E<0.55 \mathrm{~V}$ (vs SHE) by in-situ scanning tunneling microscopy. When $E \geq E_{\mathrm{pzc}}$, the potential of zero charge of the substrate, both ordered and disordered domains can be observed. Ordered domains correspond to vertically adsorbed Py molecules forming a (4 $\times 4$ ) hexagonal lattice with the nearest neighbor distance $d=0.38 \mathrm{~nm}$, and corrugation $z=0.04 \mathrm{~nm}$. The ordered adsorbate structure disappears for $E<E_{\mathrm{pzc}}$, but it is recovered several minutes after stepping $E$ backward to $E>E_{\mathrm{pzc}}$, as expected for a reversible potential-step-induced surface process. The surface concentration of adsorbed Py molecules resulting from the $(4 \times 4)$ lattice is close to $1 \times 10^{-9} \mathrm{~mol} / \mathrm{cm}^{2}$, a figure exceeding the average integral surface concentration value obtained from electroadsorption measurements. Therefore, disordered domains would be related to a mobile diluted Py adsorbate coexisting with ordered Py adsorbate domains leading to a nonhomogeneous Py adsorbate layer at the positively charged $\mathrm{Au}(111)$ terraces.
\end{abstract}

\section{Introduction}

The adsorption of pyridine (Py) on single crystal Au surfaces has been taken as a model system for studying the adsorption of organic molecules on different solid metal electrodes by electrochemical techniques. ${ }^{1}$ In neutral aqueous $0.1 \mathrm{M} \mathrm{NaClO}_{4}$ the adsorption isotherm of Py on $\mathrm{Au}(111)$ has been related to two adsorption states of $\mathrm{Py}$, depending whether the applied potential $(E)$ was more positive or negative than the potential of zero charge of the metal, $E_{\mathrm{pzc}} \cong 0.5 \mathrm{~V}$ vs SHE at $T=298 \mathrm{~K}^{2}$ When $E$ $<E_{\mathrm{pzc}}$, adsorbed Py molecules assume the parallel $\pi$-bonded orientation reaching a maximum surface concentration $\left(\Gamma_{\mathrm{m}}\right)$ close to $\Gamma_{\mathrm{m}}=0.14 \times 10^{-9} \mathrm{~mol} / \mathrm{cm}^{2}$ leading to a free energy of adsorption $\Delta G_{\text {ad }}^{\circ}<30 \mathrm{~kJ} / \mathrm{mol}$, as expected for physisorbed Py molecules. ${ }^{3}$ Otherwise, when $E \geq E_{\mathrm{pzc}}$, an increase to $\Gamma_{\mathrm{m}}=0.67 \times 10^{-9} \mathrm{~mol} / \mathrm{cm}^{2}$ and $\Delta G_{\mathrm{ad}}^{\circ}=38 \mathrm{~kJ} / \mathrm{mol}$ has been reported. This change in the values of $\Gamma_{\mathrm{m}}$ and $\Delta G_{\mathrm{ad}}^{\circ}$ is due to the reorientation of adsorbed Py molecules from a parallel configuration to a vertically N-bonded configuration. The value $\Delta G^{\circ}$ ad $=38$ $\mathrm{kJ} / \mathrm{mol}$ has been ascribed to a weakly chemisorbed state of Py on $\mathrm{Au}(111) .{ }^{1}$ Similar results have been obtained for Py adsorption on polycrystalline $\mathrm{Au}$ from aqueous $0.1 \mathrm{M}$ $\mathrm{HClO}_{4}$, at $298 \mathrm{~K}$. In this case, before the adsorption of Py on the positively charged Au surface, the pyridonium ion seems to undergo deprotonation. ${ }^{4}$

The value $\Gamma_{\mathrm{m}}=0.67 \times 10^{-9} \mathrm{~mol} / \mathrm{cm}^{2}$ mentioned above leads to the nearest neighbor average distance $d=0.5$ $\mathrm{nm}$, suggesting that the surface structure of Py on Au(111) as other organic compounds on the same metal face would involve the formation of a $(\sqrt{ } 3 \times \sqrt{ } 3)$ R30 $0^{\circ}$ hexagonal lattice. ${ }^{5}$ These conclusions, however, have not been confirmed yet by scanning probe microscopy data, par-

${ }^{\otimes}$ Abstract published in Advance ACS Abstracts, November 1, 1997.

(1) Lipkowski, J.; Stolberg, L. Adsorption of Molecules at Metal Electrode; Lipkowski, J., Ross, P. Eds.; VCH: New York, 1992; p 171, and references therein.

(2) Stolberg, L.; Morin, S.; Lipkowski, J.; Irish, D. E. J. Electroanal. Chem. 1991, 307, 241.

(3) Lipkowski, J.; Stolberg, L.; Yang, D. F.; Pettinger, B.; Mirwald, S.; Henglein, F.; Kolb, D. M. Electrochim. Acta 1994, 39, 1045.

(4) Gómez, M. M.; García, M. P.; San Fabián, J.; Vázquez, L.; Salvarezza, R. C.; Arvia, A. J. Langmuir 1997, 13, 1317.

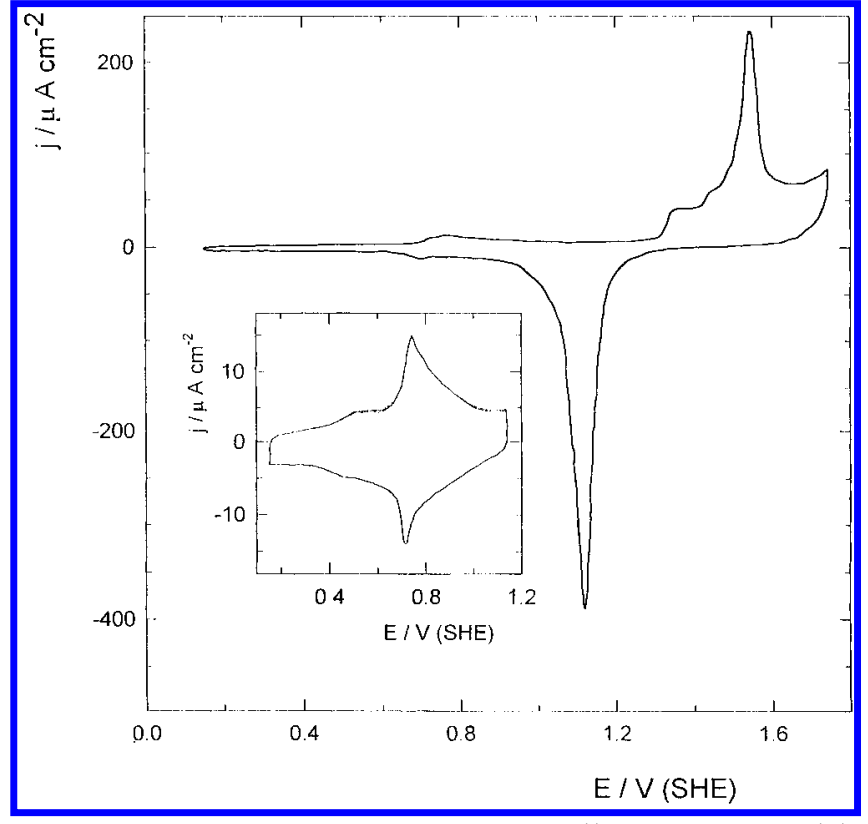

Figure 1. Apparent current density $(j)$ vs potential $(E)$ voltammogram of the Au substrate recorded between 0.15 and $1.75 \mathrm{~V}$ at $\mathrm{V}=0.05 \mathrm{~V} / \mathrm{s}$ in aqueous $0.1 \mathrm{M} \mathrm{HClO}_{4}$ at $298 \mathrm{~K}$. The inset shows the voltammogram for the same system, recorded in the double-layer region at the same $v$.

ticularly on possible Py adsorbate structures and their dynamics on $\mathrm{Au}(111)$, as has been the case for other organic molecules on the same substrate. ${ }^{5}$

This work focuses mainly on the adsorption of Py on $\mathrm{Au}(111)$ terraces from aqueous $0.1 \mathrm{M} \mathrm{HClO}_{4}+10^{-3} \mathrm{M} \mathrm{Py}$ at $298 \mathrm{~K}$ in the range of $E$ where the orientational transition of the adsorbate has been observed, following the structural changes by in-situ scanning tunneling microscopy (STM). For $E>E_{\mathrm{pzc}}$, the STM images show ordered domains, where Py molecules are oriented vertically to the $\mathrm{Au}(111)$ terraces, and disordered domains, where no molecular resolution can be obtained. In contrast

(5) Delamarche, E.; Michel, B.; Gerber, Ch.; Anselmetti, D.; Wolf, H.; Ringsdorf, H. Langmuir 1994, 10, 2869 and references therein. 


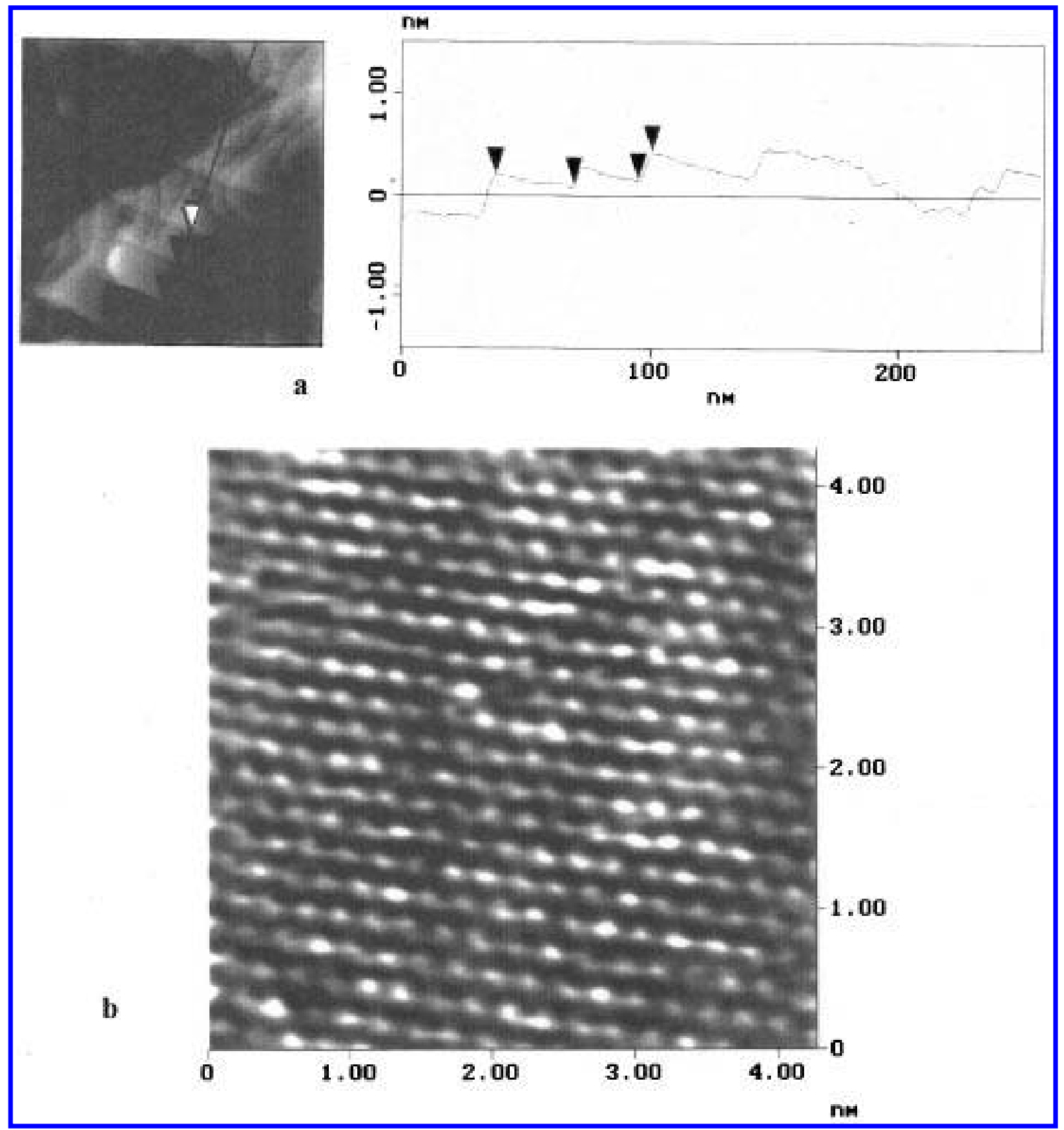

Figure 2. (a) In-situ STM image $\left(250 \times 250 \mathrm{~nm}^{2}\right)$ for the Au substrate surface in aqueous $0.1 \mathrm{M} \mathrm{HClO}_{4}$ and a cross section in the direction of the line indicated in the picture. Atomically smooth terraces and monoatomic high steps can be observed. (b) In-situ STM image $\left(4.2 \times 4.2 \mathrm{~nm}^{2}\right.$, raw data) with atomic resolution.

to the expectations, from the images of Py molecules at ordered domains, a $(4 \times 4)$ hexagonal lattice with $d=0.38$ $\mathrm{nm}$, and a corrugation $z=0.04 \mathrm{~nm}$ results. This surface structure leads to $\Gamma_{\mathrm{m}} \approx 1 \times 10^{-9} \mathrm{~mol} / \mathrm{cm}^{2}$, a figure which exceeds the value $\Gamma_{\mathrm{m}}=0.67 \times 10^{-9} \mathrm{~mol} / \mathrm{cm}^{2}$ derived from electrochemical measurements in both neutral and acid aqueous media. ${ }^{4}$ This difference indicates that for $E>$ $E_{\mathrm{pzc}}$, the ordered $(4 \times 4)$ domains coexist with others consisting of diluted domains involving mobile physisorbed Py molecules.

The potential stepping from $E>E_{\mathrm{pzc}}$ to $E<E_{\mathrm{pzc}}$ produces the gradual disappearance of ordered $\mathrm{Py}$ adsorbate domains accompanied by a partial desorption of $\mathrm{Py}$ molecules and the appearance of diluted Py adsorbate domains and uncovered $\mathrm{Au}(111)$ terraces. This potentialstep-induced Py adsorbate overlayer rearrangement behaves as a structurally reversible process for several minutes. From the analysis of the dynamics of Py adsorption on $\mathrm{Au}(111)$ in the aqueous environment, in real time, a nonuniform $\mathrm{Py}-\mathrm{Au}(111)$ site interaction energy distribution can be inferred.

\section{Experimental Section}

In situ STM images were taken using Nanoscope III Electrochemical STM equipment (Digital Instruments, Santa Barbara CA).

Runs were made at $25^{\circ} \mathrm{C}$ in the electrochemical cell consisting of the working electrode, a Pt counterelectrode, and a $\mathrm{Pd} / \mathrm{H}$ reference electrode immersed in aqueous $0.1 \mathrm{M} \mathrm{HClO}_{4}+10^{-3} \mathrm{M}$ Py. Evaporated Au films on glass (a $250 \mathrm{~nm}$ thick gold layer onto "Robax" glass, AF Berliner Glass KG, Germany) with a 2 $\mathrm{nm}$ thick chromium undercoating for better adhesion to the glass surface were employed as working electrodes. Before each experiment, these substrates were annealed under a $\mathrm{H}_{2}$ flame to obtain flat Au terraces. This type of electrode is hereafter referred to as $\mathrm{Au}$ substrate. The electrochemical cell was mounted inside a glass bell under a $\mathrm{N}_{2}$ flux to avoid the interference of $\mathrm{O}_{2}$ from the air in the electrochemical measurements. The absence of impurities was checked by recording 
current-potential profiles for the Au substrate in the acid electrolyte. Any distortion in the well-known shape of the voltammograms could be detected in these runs.

The STM imaging was performed utilizing commercial Pt-Ir tips (Digital Instruments, Santa Barbara, CA) covered by Apiezon wax to reduce faradaic current at the tip/electrolyte interface. In addition, the tip potential $\left(E_{\text {tip }}\right)$ was adjusted in the range 0.3 $\leq E_{\text {tip }} \leq 0.6 \mathrm{~V}$, i.e., in the double layer potential region of the $\mathrm{Pt}$-Ir electrode in the working solution. Typical tunneling conditions were in the ranges $0.06 \mathrm{~V} \leq E_{\text {bias }} \leq 1.5 \mathrm{~V}$ and $500 \mathrm{pA}$ $\leq I \leq 20 \mathrm{nA}$.

\section{Results}

The current density (j) vs potential $(E)$ profiles for the Au substrate immersed in aqueous $0.1 \mathrm{M} \mathrm{HClO}_{4}$, run at $V=0.05 \mathrm{~V} / \mathrm{s}$ in either the potential range $0.15 \mathrm{~V}<E<$ $1.75 \mathrm{~V}$ (Figure 1) or the double layer potential range (Figure 1 inset), resemble that already reported for Au(111).$^{6-8}$ The departure of the $j$ vs $E$ profile from that expected for a Au(111) single crystal electrode can be assigned to the relatively high density of steps at the Au substrates used in this work, as revealed by low-resolution in-situ STM images (Figure 2a) of Au electrodes under an applied potential in the range $0.5<E<0.7 \mathrm{~V}$ (see Figure 1 , inset). These images show terraces, typically 20-100 $\mathrm{nm}$ in size, with monoatomic high steps, $z=0.24 \mathrm{~nm}$. At a higher resolution, the typical hexagonal surface structure of $\mathrm{Au}(111)$ (Figure 2b) involving $d=0.29 \mathrm{~nm}$, as well as a corrugation close to $Z=0.01 \mathrm{~nm},{ }^{9}$ can be observed. In the potential range $0.5<E<0.7 \mathrm{~V}$, terraces exhibit the $(1 \times 1)$ rather than the $(\sqrt{ } 3 \times 23)$ overlayer structure reported for negatively charged flame-treated $\mathrm{Au}(111)$ surfaces in aqueous $\mathrm{HClO}_{4}{ }^{10,11}$

Similar $j$ vs $E$ voltammograms run for Au substrates in aqueous $0.1 \mathrm{M} \mathrm{HClO}_{4}+10^{-3} \mathrm{M}$ Py (Figure 3) change drastically as the threshold potential for the Au oxide monolayer formation is positively shifted, and the overall voltammogram becomes broader and strongly influenced by the adsorption of Py.

STM images of $\mathrm{Au}(111)$ terraces at $E=0.55 \mathrm{~V}$ in aqueous $0.1 \mathrm{M} \mathrm{HClO}_{4}+10^{-3} \mathrm{M}$ Py (Figure $4,0 \leq t \leq 66 \mathrm{~s}$ ) exhibit atomically flat ordered domains on terraces coexisting with other disordered domains. These images also show that the degree of ordering at the adsorbate layer appears to be constrained to $5 \times 5 \mathrm{~nm}^{2}$ domains coexisting with other domains where Py molecules cannot be resolved.

High-resolution STM images of ordered domains reveal (Figure 5a) a $(4 \times 4)$ hexagonal lattice with $d=0.38 \mathrm{~nm}$, as represented by the scheme shown in Figure $5 \mathrm{~b}$, and $z$ $=0.04 \mathrm{~nm}$. This lattice structure, which could be imaged in broad ranges of tunneling conditions, i.e., $0.06 \mathrm{~V} \leq E_{\text {bias }}$ $\leq 1.5 \mathrm{~V}$ and $500 \mathrm{pA} \leq I \leq 20 \mathrm{nA}$, can hardly be assigned to the $A u(111)$ substrate. It should be related, however, to an overlayer of adsorbed Py molecules in which the nearest neighbor distance $d=0.38 \mathrm{~nm}$ corresponds to Py molecules perpendicularly chemisorbed on $\mathrm{Au}(111)$ through the $\mathrm{N}$ atom, as has been earlier reported at $E>E_{\mathrm{pzc}}{ }^{1,3} \mathrm{It}$ should be noted that patterns $0.80 \times 0.38 \mathrm{~nm}^{2}$ in size have been observed for 2,2'-bipyridine adsorbed on $\mathrm{Au}$ (111) at $E>E_{\mathrm{pzc}}$ in neutral aqueous solutions. ${ }^{12}$

(6) Angerstein-Kozlowska, H.; Conway, B. E.; Hamelin, A.; Stoicoviciu, L. Electrochim. Acta 1996, 31, 1051

(7) Edens, G. J.; Gao, X.; Weaver, M. J. J. Electroanal. Chem. 1994, $375,357$.

(8) Hamelin, H. In Nanoscale Probes of the Solid/Liquid Interface; Gewirth, A., Siegenthaler, H., Eds.; Kluwer: Dordrecht, 1995; p 285.

(9) Behm, R. J. In Scanning Tunneling Microscopy and Related Methods; Behm, R. J., García, N., Rohrer, H., Eds.; Kluwer: Dordrecht, 1990; p 173.

(10) Gao, X.; Weaver, M. J. J. Chem. Phys. 1991, 95, 6993.

(11) Tao, N. J.; Lindsay, S. M. J. Appl. Phys. 1991, 70, 5141

(12) Cunha, F.; Tao, J. Phys. Rev. Lett. 1995, 12, 2376.

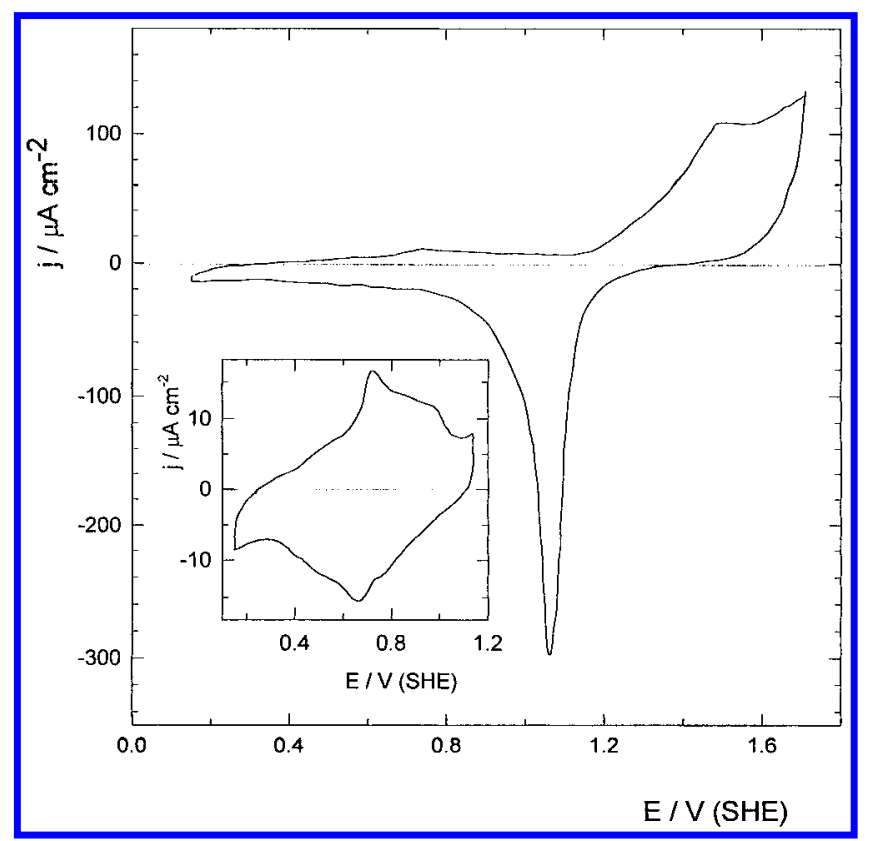

Figure 3. Apparent current density (j) vs potential (E) voltammogram of the Au substrate recorded between 0.15 and $1.75 \mathrm{~V}$ at $V=0.05 \mathrm{~V} / \mathrm{s}$ in aqueous $0.1 \mathrm{M} \mathrm{HClO}_{4}+10^{-3} \mathrm{M} \mathrm{Py}$ at $298 \mathrm{~K}$. The inset shows a voltammogram for the same system, recorded in the double layer region at the same $v$.

STM images of Py adsorbate overlayer domains on Au(111) terraces at $E=0.55 \mathrm{~V}$ also exhibit a number of depressions (black spots) forming mainly hexagonal patterns with $d=1 \mathrm{~nm}$ (Figure 6a). In some cases particles occupying these depressions are clearly visible. In principle, these patterns might be related to a moiré pattern resulting from the occupation of different Au lattice sites by Py molecules. However, a more detailed inspection of the STM images taken at different adsorption times (Figure 4) demonstrates that the black spots disappear on increasing the adsorption time, for instance, from 777 to $987 \mathrm{~s}$. Furthermore, defects with the same contrast placed at $d=0.8 \mathrm{~nm}$ can also occasionally be seen (Figure $6 c)$. These facts, which are hard to be understood in terms of a simple moiré pattern, can be explained considering that depressions are true vacancies in the adsorbate overlayer, as indicated in the scheme shown in Figure 6b. Therefore, particles which are observed inside depressions seemingly correspond to $\mathrm{Au}$ atoms from the substrate. Similar in-situSTM images with atomic defects have been reported for an adsorbed monolayer of iodine on the same $\mathrm{Au}$ substrate used in this work in $0.1 \mathrm{M} \mathrm{HClO}_{4}$ at $1.47 \mathrm{~V}$ RHE and 298 K. ${ }^{13}$ In this case, defects, which have been assigned to vacancies in the adsorbed layer caused by the partial desorption of iodine atoms, disappear when the electrode potential is stepped back to $1 \mathrm{~V}$.

The structure of Py adsorbates produced on $\mathrm{Au}(111)$ at $E=0.55 \mathrm{~V}$ is far from being frozen, as from the STM sequential imaging (Figure $4,0 \mathrm{~s} \leq t \leq 66 \mathrm{~s}$ ) it can be appreciated that boundaries between ordered domains tend to disappear progressively (Figure $4, t=0 \mathrm{~s}$ and $t=$ $28 \mathrm{~s}$, right hand side, upper region), while vacancies in the adsorbed layer are continuously produced (Figure 4, $t=28 \mathrm{~s}$ and $t=66 \mathrm{~s}$, upper, middle region) and filled (Figure $4, t=777 \mathrm{~s}$ and $t=956 \mathrm{~s}$, left hand side, upper region).

The following type of experiment demonstrates the dynamic behavior of Py adsorbates on $\mathrm{Au}(111)$ terraces at $298 \mathrm{~K}$. Starting from a Py adsorbate layer held at 0.55

(13) Batina, N.; Yamada T.; Itaya, K. Langmuir 1995, 11, 4568. 


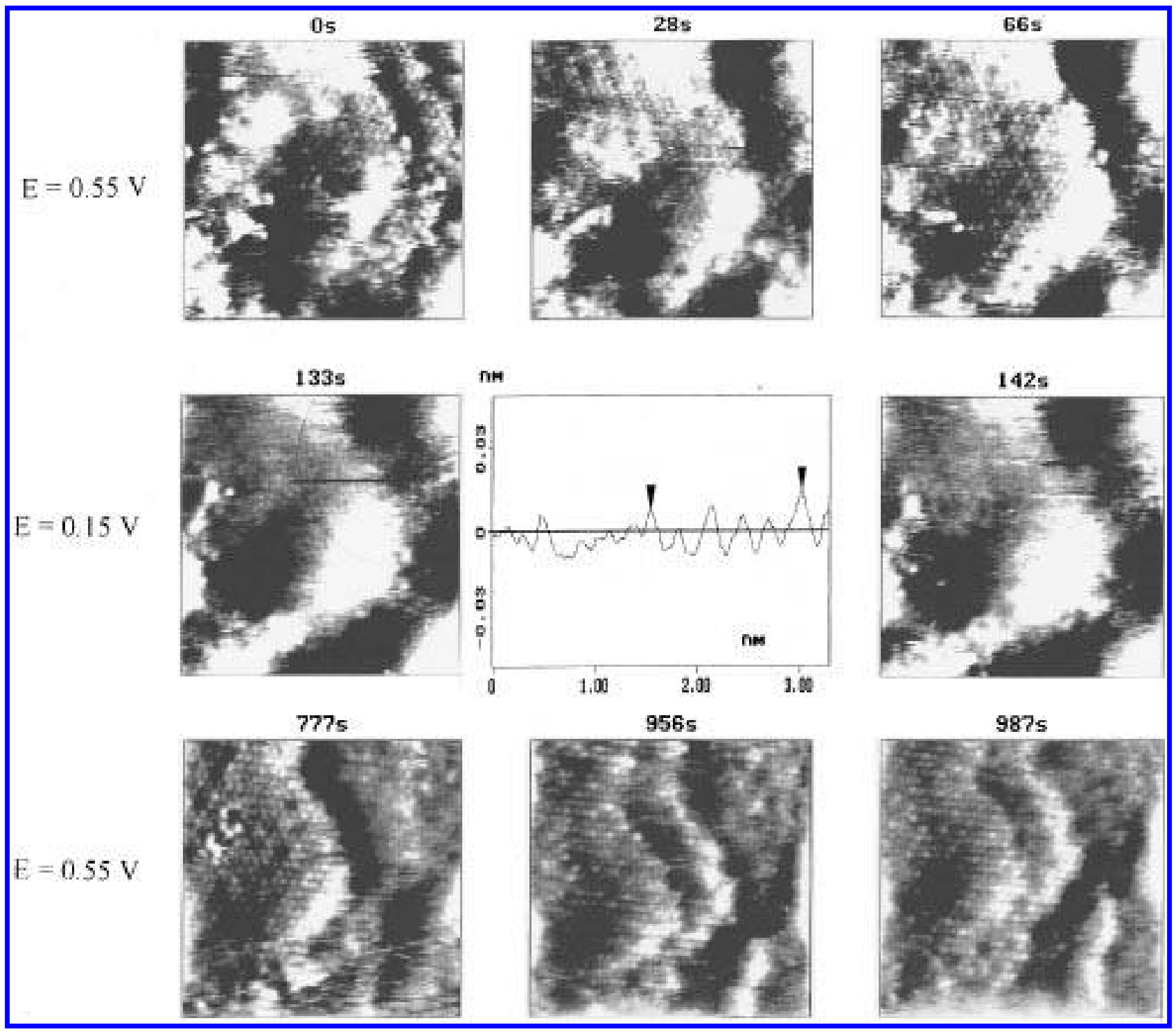

Figure 4. A sequential set of in-situ STM images $\left(10 \times 10 \mathrm{~nm}^{2}\right.$, raw data) at constant applied potential $E=0.55 \mathrm{~V}$ for $0 \mathrm{~s}<t$ $<66 \mathrm{~s}, E=0.15 \mathrm{~V}$ for $133 \mathrm{~s}<t<142 \mathrm{~s}$, and $E=0.55 \mathrm{~V}$ for $777 \mathrm{~s}<t<987 \mathrm{~s}$. Molecular resolution of the Py adsorbate at $E \geq$ $0.55 \mathrm{~V}$ can be observed for $t=0,28$, and $66 \mathrm{~s}$ and for $t>777 \mathrm{~s}$. Domains of the Au(111) substrate can be noted at $E=0.15 \mathrm{~V}$, $t=133$ and $142 \mathrm{~s}$ (middle and upper parts of the pictures). A cross section from the zoomed central region depicted in Figure 4, $t=133 \mathrm{~s}$ (solid line), is also included showing rows of atoms with $d=0.29 \mathrm{~nm}$.

$\mathrm{V}$ (Figure $4, t<66 \mathrm{~s}$ ), the stepwise change of $E$ from 0.55 to $0.15 \mathrm{~V}$ produces the gradual disappearance of the $(4 \times$ 4) hexagonal lattice in about $60 \mathrm{~s}$ (Figure 4, 133 and 142 s). In this case, the partial desorption of Py is accompanied by the appearance of rows of Au atoms (Figure $4, t=133$ $s$ and its corresponding cross section at the center of the figure). The appearance of rows of $\mathrm{Au}$ atoms after changing the potential from the adsorption to the desorption range demonstrates that the adlayer of $\mathrm{Py}$ molecules has been partially removed. ${ }^{14}$ The analysis of these STM images confirms that the Py overlayer is not rotated with respect to the underlying $\mathrm{Au}(111)$ surface. Other domains presumably covered by disordered and rather diluted Py adsorbates can be also detected. It should be noted that the order/disorder change induced by shifting $E$ from a positive to a negative value with respect to $E_{\mathrm{pzc}}$ has already been observed by in-situ STM imaging for bipyridine adsorption on $\mathrm{Au}(111) .{ }^{12}$ It has also been reported that the surface coverage by $\mathrm{Py}$ molecules is rather small with physisorbed Py molecules lying parallel to the $\mathrm{Au}(111)$ surface for $E<E_{\mathrm{pzc}} \cdot{ }^{1}$ Values of $\Delta \mathrm{G}^{\circ}$ ads lower than $30 \mathrm{~kJ} / \mathrm{mol}$ imply residence times of

(14) Gao, X.; Weaver M. J. J. Am. Chem. Soc. 1992, 114, 8544. the adsorbate at the surface of about $10^{-8} \mathrm{~s}$ for Py adsorbate on $\mathrm{Au}(111)$ terraces. ${ }^{15}$ Certainly, the residence time is much shorter than the time required for catching Py molecules by STM imaging due to the instrumental capability available at present. This fact can explain why mobile Py molecules at disordered domains cannot be easily resolved.

If the preceding experiment is continued by stepping the potential backward from 0.15 to $0.55 \mathrm{~V}$, the Py adsorbate layer slowly recovers the $(4 \times 4)$ hexagonal lattice (Figure $4, t=142 \mathrm{~s}, t=777 \mathrm{~s}, t=956 \mathrm{~s}$, and $t=$ $987 \mathrm{~s})$. Then, the growth of ordered domains from disordered adsorbate domains can be followed, as expected for a structurally reversible process undergoing for several minutes at $298 \mathrm{~K}$.

\section{Discussion}

4.1. Complexity of Py Adsorbate Patterns on Au(111) Terraces at Different Applied Potentials. The analysis of STM images provides new information about

(15) Adamsom, A. Physical Chemistry of Surfaces; J. Wiley: New York, 1990; p 593. 


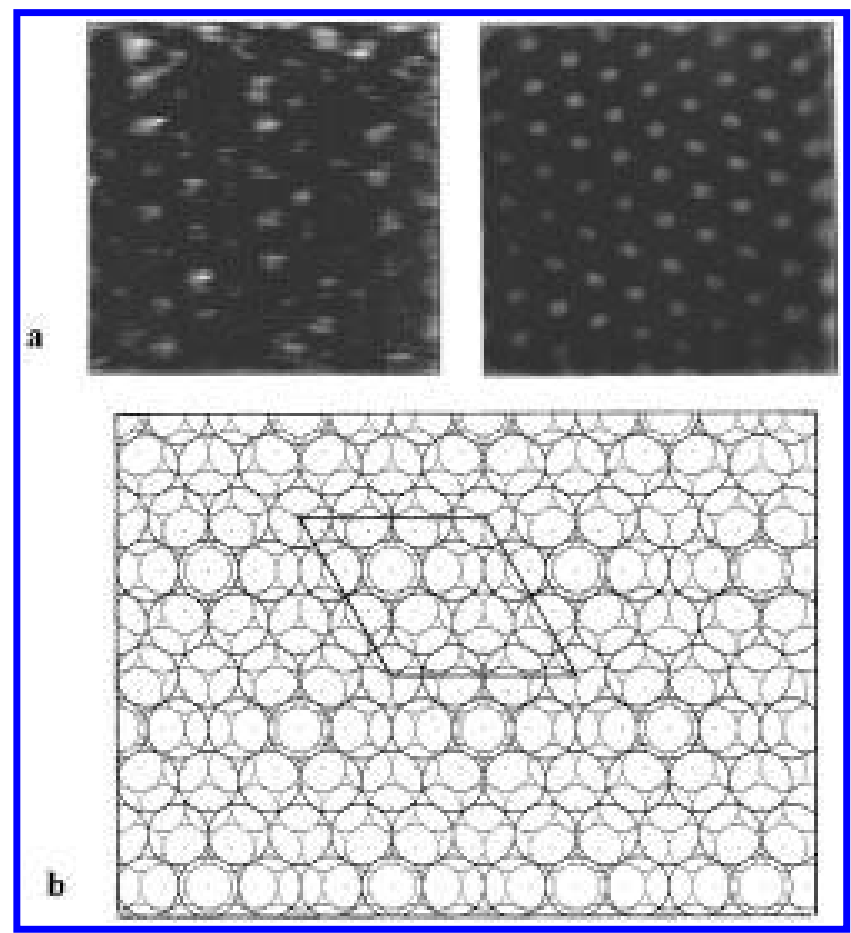

Figure 5. (a) In-situ STM (raw data and Fourier filtered) image $\left(3 \times 3 \mathrm{~nm}^{2}\right)$ of an ordered domain showing the hexagonal array of Py molecules. (b) Scheme of the $(4 \times 4)$ Py overlayer on Au(111). Large circles denote Py molecules and the small ones correspond to $\mathrm{Au}$ atoms.

the complex structure of Py adsorbates on $\mathrm{Au}(111)$ terraces and adsorbate overlayer rearragements induced by stepping the potential across the value of $E_{\mathrm{pzc}}$.

The adsorption of Py on smooth $\mathrm{Au}(111)$ terraces at $E$ $\geq E_{\mathrm{pzc}}$ results in the formation of ordered domains, typically $5 \times 5 \mathrm{~nm}^{2}$ in size, exhibiting a close-packed $(4 \times 4)$ hexagonal lattice of vertically adsorbed molecules (Figure 5) coexisting with disordered domains (Figure 4). Furthermore, a relatively large number of defective sites in the $(4 \times 4)$ hexagonal lattice can also be observed. These defects form a hexagonal commensurate pattern on Au(111) terraces with $d=1 \mathrm{~nm}$ (Figure 6a,b), which coincides with the position of Au atoms where Py molecules would be adsorbed atop. In fact, the coincidence between the scheme and the STM image is remarkable. The cross section analysis of these patterns (Figure 6a,b) indicates the location of the Py adsorbate at hollow positions at a distance $\sqrt{ } 3 \times 0.38 \mathrm{~nm}$ (arrows A) and the location of vacancies at $\sqrt{ } 3 \times 1 \mathrm{~nm}$ coinciding with atop adsorption sites for Py (arrows B). Therefore, the high density of these defective sites allows us to conclude that when Py molecules are adsorbed atop, comprising a lower coordination at the surface, the value of $\Delta G^{\circ}$ ads becomes less negative than that for Py adsorbates at other Au lattice locations. Therefore, Py molecules adsorbed atop would be more easily desorbed from the substrate.

The preceding description is consistent with the fact that after the potential reversal from $E<E_{\mathrm{pzc}}$ to $E>E_{\mathrm{pzc}}$ the number of vacancies present in the adsorbed layer tends to decrease slowly as the close-packed $(4 \times 4)$ lattice is formed (Figure 4,777 $\mathrm{s} \leq t \leq 987 \mathrm{~s}$ ). At this intermediate stage, it is reasonable to admit that most vacancies correspond to sites for adsorption of Py atop as $d \approx 1 \mathrm{~nm}$. However, a zoom of these STM images also shows vacancies separated by $d \approx 0.8 \mathrm{~nm}$ (Figure 6c), a distance coinciding with that assigned to the nearest neighbour adsorbed Py molecules at near bridge positions. Therefore, for $E>E_{\mathrm{pzc}}$, Py molecules are firstly adsorbed at hollow positions, then at the near bridge positions, and finally at

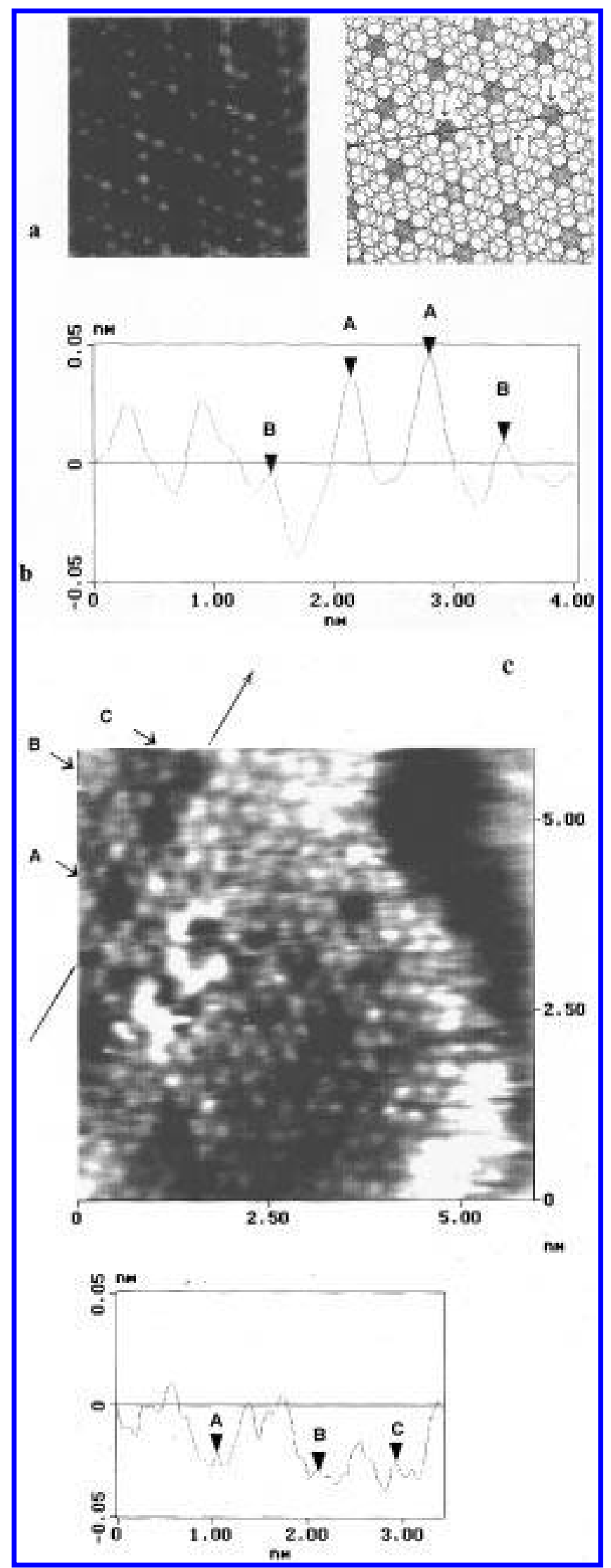

Figure 6. (a) In-situ STM $\left(4 \times 4 \mathrm{~nm}^{2}\right.$, Fourier filtered) image of the $(4 \times 4)$ adsorbed Py lattice including the hexagonal pattern of defects. (b) A cross section resulting from the image shown in (a). Atop and hollow Py adsorbate distances are indicated by arrows B and A, respectively. The scheme of the Py adsorbate layer indicated in a) accounts for the features of the cross section depicted in (b). (c) In-situ STM image $\left(6 \times 6 \mathrm{~nm}^{2}\right.$, raw data) and a cross section showing defects $A, B$, and $C$ in the Py adsorbate layer at distances $d(\mathrm{~A}-\mathrm{B})=1 \mathrm{~nm}$ and $d(\mathrm{~B}-\mathrm{C})=0.8 \mathrm{~nm}$. 
atop sites. This fact suggests that the value of $\Delta G^{0}$ ad for $\mathrm{Py}$ adsorption on $\mathrm{Au}(111)$ is site-dependent increasing negatively in the order atop $<$ near bridge $<$ hollow positions.

4.2. Local and Global Surface Coverage of Au(111) Terraces by Py Adsorbates in the Aqueous Solution. The occurrence of the $(4 \times 4)$ pattern for Py molecules adsorbed on $\mathrm{Au}(111)$ for $E>E_{\mathrm{pzc}}$ implies $\Gamma_{\mathrm{m}}=$ $1.32 \times 10^{-9} \mathrm{~mol} / \mathrm{cm}^{2}$. This figure decreases to $\Gamma_{\mathrm{m}}=1 \times$ $10^{-9} \mathrm{~mol} / \mathrm{cm}^{2}$ when depression-containing patterns are formed. Both figures, however, exceed $\Gamma_{\mathrm{m}}=0.67 \times 10^{-9}$ $\mathrm{mol} / \mathrm{cm}^{2}$ reported for $\mathrm{Py}$ adsorption at $E>E_{\mathrm{pzc}}$ from electroadsorption measurements at $298 \mathrm{~K}$ either on $\mathrm{Au}$ (111) from aqueous $0.1 \mathrm{M} \mathrm{NaClO}_{4}^{1,3}$ or polycrystalline $\mathrm{Au}$ from aqueous $0.1 \mathrm{M} \mathrm{HClO}_{4}{ }^{4}$ The value $\Gamma_{\mathrm{m}}=0.67 \times 10^{-9}$ $\mathrm{mol} / \mathrm{cm}^{2}$ implies $d=0.5 \mathrm{~nm}$ for the Py-Py distance at the adsorbate lattice. This value of $d$ turns out to be close to that derived from the $(\sqrt{ } 3 \times \sqrt{ } 3)$ R30 ${ }^{\circ}$ lattice formed on the same substrate by a certain number of organic molecules including short and long aliphatic chain thiols. ${ }^{5}$ The presence of the $(4 \times 4)$ hexagonal structure is to some extent surprising specially considering that related compounds as bipyridine exhibit a quite different structural arrangement. ${ }^{12}$ However, even based on thermodynamic $\Gamma_{\mathrm{m}}$ data, a more dense packing should be expected for Py $\left(\Gamma_{\mathrm{m}}=0.67 \times 10^{-9} \mathrm{~mol} / \mathrm{cm}^{2}\right)$ than for bipyridine $\left(\Gamma_{\mathrm{m}}=0.5\right.$ $\left.\times 10^{-9} \mathrm{~mol} / \mathrm{cm}^{2}\right)$. Such a high packing density of the Py adsorbed layer suggests that $\mathrm{Py}-\mathrm{Py}$ attractive interactions play a key role in the stability of ordered Py adsorbate domains.

The apparent discrepancy in the values of $\Gamma_{m}$ obtained from STM and electrochemical techniques for Py adsorption is not surprising if one takes into account that they were derived, in one case, from very local measurements and, in the other, from integral electrochemical measurements. In contrast to bipyridine adsorption on $\mathrm{Au}(111)$ at $298 \mathrm{~K}$, where a coincidence between the values of $\Gamma_{\mathrm{m}}$ resulting from STM imaging and thermodynamic data has been found, ${ }^{12}$ the type of discrepancy described above has also been observed for uracil molecules on $\mathrm{Au}(111)$ surfaces. ${ }^{16,17}$ In this case, STM images reveal hexagonal patterns with typical distances of $0.4 \mathrm{~nm}^{16}$ leading to a higher value of $\Gamma$ than that derived from electroadsorption measurements. ${ }^{17}$

(16) Holzle, M. H.; Wandlowski, Th.; Kolb, D. M. Surf. Sci. 1995, $335,281$.

(17) Wandlowski, T. H.; Ocko, B. M.; Magnussen, O. M.;Wu, S.; Lipkowski, J. J. Electroanal. Chem. 1996, 409, 155.
From the preceding description and values of $\Gamma_{\mathrm{m}}$, at $E$ $>E_{\mathrm{pzc}}$, it can be inferred that Py adsorption on $\mathrm{Au}(111)$ terraces consists of a heterogeneous adsorbate layer involving ordered domains, where Py molecules are vertically oriented with respect to the substrate plane, and disordered domains, where Py molecules are placed parallel to the substrate. The presence of domains with no molecular or Au atom resolution surrounding ordered domains (Figure 4, $0 \mathrm{~s} \leq t \leq 66 \mathrm{~s}$, and Figure $4, t=142$ $\mathrm{s}, t=777 \mathrm{~s}, t=956 \mathrm{~s}$, and $t=987 \mathrm{~s})$ supports this scenario for Py adsorption on Au(111) terraces at these $E$ values.

The growth of ordered domains at the expense of disordered domains, as seen in Figure $4, t>777 \mathrm{~s}$, clearly starts at step edges and continues across terraces to reach the steps of the upper terraces. The appearance of a large number of defects at ordered domains supports the idea that they grow at the expense of disordered domains.

\section{Conclusions}

Py adsorbates at $\mathrm{Au}(111)$ terraces in aqueous $0.1 \mathrm{M}$ $\mathrm{HClO}_{4}+10^{-3} \mathrm{Py}, 298 \mathrm{~K}$, and $E>E_{\mathrm{pzc}}$ form ordered $(4 \times$ 4) domains, where molecules are vertically placed, and disordered domains, where mobile, physisorbed molecules are parallel to the $\mathrm{Au}(111)$ terrace. The existence of these distinguishable domains is consistent with the value $\Gamma_{\mathrm{m}}$ $=0.67 \times 10^{-9} \mathrm{~mol} / \mathrm{cm}^{2}$ resulting from electrochemical measurements.

The change of the applied potential from $E>E_{\mathrm{pzc}}$ to $E$ $<E_{\mathrm{pzc}}$ produces the partial desorption of Py molecules from the ordered domains, yielding diluted adsorbate domains and uncovered $\mathrm{Au}(111)$ terrace sites.

The dynamics of Py adsorbates at a $\mathrm{Au}(111)$ surface, followed by stepping the potential from $E>E_{\mathrm{pzc}}$ to $E<$ $E_{\mathrm{pzc}}$ and vice versa, indicates a structurally reversible surface rearrangement of adsorbates.

The analysis of $\mathrm{Py}$ adsorption patterns for $E>E_{\mathrm{pzc}}$ provides information about the local characteristics of $\mathrm{Py}$ adsorption on a $\mathrm{Au}(111)$ terrace, suggesting the existence of different values of the adsorption energy for $\mathrm{Py}$ molecules adsorbed atop, at near bridge, and at hollow sites.

Acknowledgment. This work was financially supported by the Consejo Nacional de Investigaciones Científicas y Técnicas (CONICET) of Argentina, the Comisión de Investigaciones Científicas de la Provincia de Buenos Aires (CIC), and Fundación “Antorchas” (Argentina).

LA970417R 